\title{
Psychosocial aspects of living with diabetes mellitus in promoting self-care
}

\author{
Aspectos psicossociais do viver com diabetes Mellitus na promoção do autocuidado
}

Sumaya Giarola Cecilio ${ }^{1}$, Célia Luciana Guedes Barbosa Brasil ${ }^{1}$, Camila Pacheco Vilaça ${ }^{1}$, Samara Mariana Ferreira da Silva ${ }^{1}$, Elisângela da Cruz Vargas ${ }^{1}$, Heloísa de Carvalho Torres ${ }^{1}$

Objective: to understand the psychosocial aspects of living with diabetes mellitus. Methods: a study qualitative, made with 16 patients with diabetes mellitus type 2 . The data were collected through telephone calls using a semi-structured script approaching the care in health, barriers and family support, analyzed by Bardin's content. Results: two categories emerged related to the practice of self-care: Self-care, containing three sub-categories: diet, physical activity and emotional aspects and support networks. Conclusion: the results of the study showed the importance of understanding the emotional aspects and attitudes of patients regarding educational practices for self care, in order to establish strategies to prevent and control the disease.

Descriptors: Health Education; Self Care; Diabetes Mellitus; Telemedicine.

Objetivo: compreender os aspectos psicossociais em viver com a condição do diabetes Mellitus. Métodos: estudo qualitativo, realizado com 16 usuarios com diabetes Mellitus tipo 2. Os dados foram coletados mediante ligação telefônica utilizando-se um roteiro semiestruturado abordando o cuidado em saúde, barreiras e apoio familiar, analisados por conteúdo de Bardin. Resultados: emergiram duas categorias relacionadas à prática de autocuidado: Cuidar de si, contendo três subcategorias: alimentação, atividade física e aspectos emocionais e Redes de apoio. Conclusão: os resultados do estudo mostraram a importância de compreender os aspectos emocionais e as atitudes dos usuários em realção as práticas educativas para o autocuidado, de forma a estabelecer estratégias de prevenção e controle da doença.

Descritores: Educação em Saúde; Autocuidado; Diabetes Mellitus; Telemedicina.

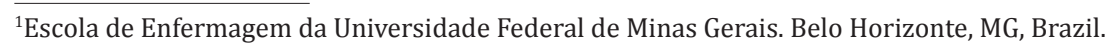




\section{Introduction}

Monitoring and intervening in health in order to promote the practice of self-care of the patients with diabetes is an action that should include the subjective and emotional aspects, in order to profoundly understand the factors that interfere in the treatment. The initial steps for the process of construction of selfcare results from exploration and analysis of feelings, leading to reflection and questioning of the reality ${ }^{(1-3)}$.

Among the different approaches that can be used to understand the issues related to self-care in diabetes, there is the use of phone calls, which aims at offering assistance to patients of the health service, building a bond and monitoring health conditions as well as providing continuity of contact, for the prevention of chronic complications ${ }^{(4-5)}$.

The telephone call is a feasible technology of care and of low cost, which allows, in a short time, the health professional to have access to the context of life of the patients, their anguishes, anxieties and difficulties $^{(4)}$. Systematized actions to provide the means to intervene with the patients, groups, and expand the ideas for the community are considered a technology of care, aiming at promoting well-being in health ${ }^{(6)}$.

Some studies indicate the importance of telephone calls in the creation of bond and highlight that the patient feels welcomed and valued, thus reflecting on their self-esteem and psychosocial aspects. Emotions, negative feelings, fear, anguish and anxiety are factors which are present in the treatment of diabetes and that are sometimes neglected by Health professionals. The effectiveness of care concerning the diabetes is associated not only to everyday choices but above all, the ability to control the emotional issues that are directly linked to the outcome of the treatment ${ }^{(5-7)}$.

As such, the aim of this study is to analyze the psychosocial aspects of living and performing practices of self-care in diabetes through telephone calls.

\section{Methods}

It is a descriptive and exploratory study, of qualitative nature, made with 16 patients with diabetes mellitus type 2 in eight health centers in Belo Horizonte, MG, Brazil. The criteria of inclusion were patients between 20 and 75 years, with time of diagnosis of diabetes less than twenty years and not having chronic complications, such as diabetic retionopatia, neuropathies, heart disease and kidney diseases.

Data were collected from March to September 2014, through telephone calls with an average duration of 40 minutes, made by nurses, having as objective to know the psychosocial aspects of living with diabetes. Previously, the health professionals participated in trainings in order to have qualified listening and dialogic practice ${ }^{(8)}$.

A semi-structured script to guide the data collection was used, which contained the following questions: What is your greatest difficulty to control diabetes ?; talk me more about this difficulty in your everyday life; Give example (s) of a situation that happened to you because of this difficulty; How do you feel about this situation of having to take care of your health (control and take care of the disease)?

In the course of the telephone calls, the speeches were being manually recorded and also through recordings, and these systematized and categorized materials in databases for analysis through Bardin's referentials, which proposes three stages of thematic analysis: Pre analysis; Exploration of material and treatment of results: inference and interpretation ${ }^{(9)}$. So it was possible to find groups of meaning in the analysis of the speeches of the patients and find two thematic categories: Self-care and support networks.

The study met the proposed saturation criteria for qualitative research. The point of saturation depends indirectly on the theoretical background adopted by the researcher and the research objectives, in addition to the homogeneity of the study population ${ }^{(10)}$. Saturation was achieved on the 
data from the 16th telephone call, from the moment that reports became similar with the same response content.

The study complied with the formal requirements contained in the national and international regulatory standards for research involving human beings.

\section{Results}

Of the 16 patients with diabetes mellitus type 2 , the majority $(73.2 \%)$ lived with the condition for over five years with 60 years of age or more $(78.0 \%)$, female (66.0\%), residents in Belo Horizonte, capital of Minas Gerais. Among them, 63.0\% reported monthly income from one to two minimum wages (minimum wage in 2014: $R \$ 724,00$, referring to the dollar in 2014: U \$ 177.50) $62.0 \%$ had the less than 9 years of study.

Through the investigation of the psychosocial aspects of living with diabetes, two categories related to the practice of self care were identified: Taking care of yourself and support networks.

Taking care of oneself in the scope of diabetes is not a simple task for most of the study subjects. It is presented as a practice involved in many verbs "have" and few verbs "want", which refers to an idea of obligation having to take care of their own health.

Changing the behavior in health and having a need to control the actions of care generates some obstacles in the process, which we call barriers. Due to the multiplicity of barriers mentioned by the patients, it was decided to present them in sub-categories: physical activity, nutrition and emotional aspects. The emotional aspects are present and are associated to all self-care practices, then, even if a sub-category has been created for the feelings, they are present in all the others.

\section{Subcategory 1- Physical Activity}

The first topic, difficulty in performing physical activities, was attributed to the lack of organization of routine and lack of courage to exercise such practice. It is possible to observe that patients are aware of the benefits of regular physical activity, however, they have not yet incorporated that as a goal for self-care: Lack of discipline to perform physical activity, this is what most disturbs me (U1); I'm really trying to get working out (U2); I do not have this purpose: tomorrow I will do it! I'm really discouraged. One thing that I feel I can do and should do is physical activity (U3).

A discouragement in the statements was observed, which goes along with a negative feeling associated to the routine of care in diabetes: $A$ month ago I was in a center of treatment of the body by a request of my daughter. But I did not return because I was lazy. I feel very discouraged (U5); ... I begin a routine of physical activity, such as walking in a month. However, next month I'm already unmotivated again (U4).

\section{Subcategory 2 - Nutrition}

Another aspect presented consists in the difficulty in eating healthily. Patients associate this question to financial factors where the high cost of the diet impairs to follow the feeding plan: Due to the lack of money I can not keep buying dietary things because they are more expensive (U11). They resent the fact of having to follow a diet, expressing the difficulty to develop self-control and not eating as they would like: Bread, is the food that I like! However, because I know it's not suitable for me, I switch the types of bread: one day, wholewheat bread, another day, regular bread (U7); ... Eating impairs the practice of self-care. For example, sometimes even knowing that I have to decrease the carbs, I can not manage and end up eating all the same (U4).

Few patients are aware that the eating standard harms them and can bring more severe damages to their health, but even so they keep on exaggerating. Some days I ingest an excessive amount of sweets and end up feeling very bad (U10); I am wrong concerning eating. Instead of making a healthy choice, I choose food with a lot of saturated fat (U13); I remain a long time without eating, I don't split the meals. I believe this is lack of discipline in my life (U12).

As mentioned at the beginning of the category, the emotional factors are present and they interfere 
in following an ideal eating plan to keep the glucose controlled: Sometimes I eat badly because I am lazy to prepare something healthy, such as vegetables and green leaves. But I really feel very discouraged and it disturbs me to develop certain activities (U15). Still, the need to make the diet also interferes in the social activities of the patient: I do not feel like going out because when I go to a birthday party, I see food and I really want to eat them, but I know I can't! So, I want to eat and I can't, and that is my biggest difficulty (U11).

\section{Subcategory 3 - Emotional aspects}

Due to the chronicity of diabetes, the "being" and "living" with diabetes becomes a great and arduous path in health care, generating intense emotional reactions, which makes it necessary to go beyond the conventional barriers and understand that the limitations caused by emotional aspects are responsible for the results of the treatment.

This fact can be evidenced in the statements of the patients: Follow the diet, do physical exercise. Following the diet is, for example, not eating the things that I can't, avoid sweet, avoid pasta, right ... But I'll be honest, the emotional side will not let me go on, I can't! (U6).

Other patients express their anger and sadness of having to live with the most severe form of diabetes, refusing to follow the treatment: Some things are not within us... that stage was very difficult and I did the exams and I said to the doctor, you know what, I want to die, you do not need to increase medication because the pain is too much, it is very strong and I do not know if I can stand that (U7); But there are times when I stop and think: am I the only rebel? (U10).

When the patient feels resilient to his condition of health and have sufficient motivation to take care of himself, the impacts on quality of life and the results in the treatment are better: I even think it's nice to, like, have a responsibility to take care. So I think it's nice! I take care of myself, I accept and it's okay (U10).

However, most expressed anxiety and depressive symptoms facing the treatment, feeling unmotivated: So, I get anxious and start to eat the things I can't (U1); Ah ... to apply [insulin] every day is not easy, is it? (U15); Oh, my end will be very sad, right (U16); And because sometimes I plan here ...then I discouraged, I don't do it (U11).

Many fear to have stories with tragic endings, such as relatives or close friends, demonstrating a fear of chronic complications: Now I have a historic record in the family with diabetes, my brother died, an uncle amputated a leg, a niece, that studiedl nursing, became suddenly ill, her glucose reached $600 \mathrm{mg} / \mathrm{dl}$ (U13).

\section{Category 2: Support networks}

Family members were mentioned by the patients as the main supporters in health care. They reported support for the feeding plan, helping them to feed in a fractional manner, cooperating in the physical activities and encouraging them to adopt healthy habits of life, and remind them about the medication schedules.

Spouses, especially female, were mentioned fondly by patients, who highlighted their relationship: It's the same as walking, if I have to go alone, I get discouraged. She [wife] is my companion for all (U9); And she charges me too much, she comes and asks if I walked. She keeps on trying to make me go to the gym, but I don't want to go to the gym much (U7).

The Health professionals were mentioned as partners in the process, helping in coping with the disease and performing educational practices in health. Still, the patients highlighted the bond of friendship and empathy developed with professionals, which also leads to motivation for the change of the lifestyle and self-care: I can't wait for the meetings Igroups of education in health] to be back, once there I had goals to achieve, I felt more excited (U10); With your help, I started eating properly, not eating much salt, nor much fat, always telling myself to walk and take medicine properly, everything is going well, thank God (U12).

\section{Discussion}

The telephone call is a recent technology of health care and it is proposed to continue the process begun in educational programs for patients with diabetes, aiming at building self-care and working with the issues that hinder this process ${ }^{(3,5-6)}$. In this 
study, the psychosocial aspects of living with diabetes were analyzed, strengthening the practice of self-care and revealing their main barriers.

The population of $78.0 \%$ of elderly in the study was consistent with the educational proposal, since when there is a prevalence of young people and adults, it is possible to choose the use of technologies aimed at more virtual communication, such as text messages, use of applications and e-mails. However, for older adults, instructions given face to face and home phone, are more suitable ${ }^{(11)}$.

Despite new treatments and new therapeutic options, most patients still fail to achieve adequate levels of blood glucose and they suffer from such condition. Studies show that the challenges faced by patients with diabetes include psychosocial aspects, as these can become the real barriers to achieve good health outcomes. There is no psychological support and mainly no exploration of such aspects by the health professionals ${ }^{(12)}$.

Studies which try to go into the psychosocial aspects in living with diabetes, and in researching the barriers for self-care highlight aspects such as motivation, depression and the importance of support networks, as reported in the results of this study. Motivation is shown as something that can be extrinsic or intrinsic: the former offered by the health team or family members and the latter as having the greatest weight to success in the treatment of diabetes. Patients who are more secure of their abilities can manage to change their attitudes more easily and with less suffering ${ }^{(13)}$.

That became clear in the Emotional Aspects category, when the patient feels resilient to his health condition, who starts to have a greater motivation to take care of himself. However, when that is not present, it is presented as an important barrier to be worked on, especially by the health professionals, who must take an altruistic approach to the patients, enabling and helping in exploring their feelings, needs and values related to care health ${ }^{(14)}$.
Besides the motivation (or lack of it), the presence of negative feelings associated to diabetes suggest the presence of depression, commonly observed in patients with diabetes which can negatively affect the way patients take care of themselves. It is important to highlight that the patients who are depressed show symptoms such as the loss of interest for self-care, tiredness and low self esteem $^{(14-15)}$.

The barriers concerning eating or physical activity usually prevents these patients to be active and to feel pleasure to take care of themselves, they are the negative feelings associated to the need to change habits $^{(15)}$. The stress generated by the obligation to follow an eating pattern was unanimous in the statements of the patients of this study, corroborating with the findings in the literature that the fact of not being able to change the behavior is stressful and generates negative feelings ${ }^{(12)}$.

The sadness and the feeling of revolt were also observed, which is perceived as a reaction to the diagnosis and treatment. Besides that, fear is present in the emotional aspects category, as a response to the disbelief in the treatment and in the aprehesion concerning the emerging of other diseases or even death resulting from diabetes complications ${ }^{(12)}$, which comes to present dichotomy in the practice of care: while the patients present fear of their condition and fear of the future, they meant to take care of themselves as they would like. There is fear of complications, however, there is resistance to care.

It is in this scenario that the supporting networks can help. The presence of the health professionals in the statements of the patients expresses the continious need for information and support. The educational practice when is reflective, open to dialogue and to solving of problems, shows to be liable to present good results, both in glucose levels and in the self-confidence and satisfaction with the treatment ${ }^{(15-16)}$. It is important that professionals seek to understand the particularities of each patient, 
so that, the educational actions are assertive in order to fullfil the needs of the patients, based on the construction of realistic targets, from the difficulties highlighted by the patients and not predetermined by professionals or health care ${ }^{(17)}$.

The actions of companions, family members and close friends have a positive impact on the treatment. Companions who are willing to provide support, assist the practice of physical activity and help the patients to have balanced meals, help to promote better results from the time that they incentivate and assist a healthy lifestyle ${ }^{(16)}$. In the Supporting Network category, those patients who counted on the continued support of partners and family members, manage to reach theirr goals, such as physical activity, and felt more motivated, which generates the reflection on the need to educate not only the patients, but above all, their companions and members of everyday life ${ }^{(16)}$.

In short, living with diabetes is to live in search of reasons for their condition, is to have the need to adopt a position in relation to their care and have a more autonomous and reflective attitude towards their empasses. The practice of self-care is a long gradual process, which needs to be constantly evaluated with daily doses of motivation. It is necessary to try to balance psychosocial aspects of diabetes by harmonizing the negative and positive poles, so that the perception of health does not deteriorate into a counterproductive care.

So, the role of education in health is reaffirmed in its multiple strategies such as telephone calls as a possibility to providesupport, build knowledge, change unhealthy habits, prevent chronic complications and mainly work the aspects that influence the quality of life of the patients ${ }^{(18-19)}$.

However, it is necessary that this contact is continuous monitored, so that the health professional can guide the educational activities to encourage the practice of self-care, and anticipate the inherent complications to diabetes. Thus, the telephone call becomes a viable proposition since, it proposes the establishment of goals, updates the knowledge regarding health care, establishes the creation of patient/health profession bond, leading to a better perspective of care $^{(3,5-6)}$.

As a limitation of the study, there is the location of the sampling of the study, with all the patients belonging to the same region. It is necessary to know the barriers related to self-care in different cultures and locations.

\section{Final Considerations}

Whereas psychosocial factors can add more complexity to diabetes self-care, holistic approaches that encompass people with diabetes and their families need to be adopted. The psychosocial factors strongly influence the ability of the patient to incorporate practices of self-care and to change their behavior concerning health.

For such, education on diabetes should transmit key information that will help the patient understand the emotional aspects and attitudes regarding educational practices for self-care, thus promoting a healthy lifestyle.

In this study, the main difficulties presented by the patients were related to physical activity, nutrition and emotional aspects. The latter being present and associated to all other aspecta. This fact confirms the need to analyze and understand the impact of the psychosocial aspects, above all the negative feelings and attitudes on the practices of self-care in diabetes.

Understanding the characteristics of the patient with diabetes is a urgent need for the Health professionals, who can explore them through educational practices such as telephone intervention calls, providing the construction of the bond and the corresponsibility of care.

\section{Acknowledgement}

The Fundação de Amparo a Pesquisa de Minas Gerias - Process: APQ-03598-12. 


\section{Collaborations}

Cecilio SG contributed to the design of the project, analysis of the results and final approval of the version to be published. Brazil CLGB, Vilaca CP, Silva SMF and Vargas EC contributed in the collection of data, writing of the article and. Torres HC contributed in the writing of the article, relevant critical revision of the intellectual content and final approval of the version to be published.

\section{References}

1. Tang TS, Funnell MM, Brown MB, Kurlander JE. Self-management support in "real-world" settings: an empowerment-based intervention. Patient Educ Couns. 2010; 79(2):178-84.

2. Zoffmann V, Kirkevold M. Realizing empowerment in difficult diabetes care: a guided selfdetermination intervention. Qual Health Res. 2012; 22(1):103-18.

3. Goodarzi M, Ebrahimzadeh I, Rabi A, Saedipoor $B$, Jafarabadi MA. Impact of distance education via mobile phone text messaging on knowledge, attitude, practice and self efficacy of patients with type 2 diabetes mellitus in Iran. J Diabetes Metab Disord [internet]. 2012 [cited 2015 Mar. 13];11(1):10. Available from: http://www.ncbi. nlm.nih.gov/pmc/articles/PMC3598175/

4. Gamboa M, Perez S, Vrotsou K, Arbonies OJC, Del Campo PE, Ochoa RGL, et al. Impact of a self-care education programme on patients with type 2 diabetes in primary care in the Basque Country. BMC Public Health [internet]. 2013 [cited 2015 Mar. 13];13:521. Available from: http://www. ncbi.nlm.nih.gov/pmc/articles/PMC3680082/

5. Torres H, Reis IA, Roque C, Faria P. Monitoramento telefônico como estrategia educativa para o autocuidado das pessoas com diabetes na atenção primária. Cienc Enferm. 2013; 1(1):95-105.

6. Beradinelli LMM, Guedes NAC, Ramos JP, Silva MGN. Tecnologia educacional como estratégia de empoderamento de pessoas com enfermidades crônicas. Rev Enferm UERJ. 2014; 22(5):603-9.
7. Wu JJ, Sung HC, Chang AM, Atherton J, Kostner K, Courtney M, etal. Protocol for a randomised blocked design study using telephone and text-messaging to support cardiac patients with diabetes: a cross cultural international collaborative project. BMC Health Serv Res [internet]. 2013 [cited 2015 Mar. 13]; 13(1):402. Available from: http://www.ncbi. nlm.nih.gov/pmc/articles/PMC3852414/

8. Salci M, Maceno P, Rozza S. Health education and its theoretical perspectives: a few reflections. Texto Contexto Enferm. 2013; 22(1):224-30.

9. Bardin L. Análise de conteúdo. Lisboa: Edições 70; 2011.

10. Fontanella BJB, Ricas J, Turato ER. Amostragem por saturação em pesquisas qualitativas em saúde: contribuições teóricas. Cad Saúde Pública. 2008; 24(1):17-27.

11. Mulvaney SA. Improving patient problem solving to reduce barriers to diabetes self management. Clinical Diab. 2009; 27(3):99-104.

12. Nicolucci A, Burns KK, Holt RIG, Comaschi M, Hermanns N, Ishii H, et al. Research: Educational and Psychological Issues Diabetes Attitudes, Wishes and Needs second study (DAWN2TM): Cross diabeteswith diabetes. Diabet Med. 2013; 30(7):767-77.

13. Ahola AJ, Groop PH. Barriers to self-management of diabetes. Diabet Med. 2013; 30(4):413-20.

14. Lopes AAF. Cuidado e Empoderamento: a construção do sujeito responsável por sua saúde na experiência do diabetes. Saúde Soc. 2015; 24(2):486-500.

15. Ludman EJ, Peterson D, Katon WJ, Lin EH, Von Korff $\mathrm{M}$, Ciechanowski P, et al. Improving confidence for self care in patients with depression and chronic illnesses. Behav Med [internet]. 2013[cited 2015 Mar. 13]; 39:1-6. Available from: http://www. ncbi.nlm.nih.gov/pmc/articles/PMC3628828/

16. Van Puffelen AL, Rijken M, Schellevis FG. Living with diabetes: a group-based self-management support programme for T2DM patients in the early phases of illness and their partners, study protocol of a randomised controlled trial. BMC Health Serv Res [internet]. 2014 [cited 2015 Mar. 13]; 14:144. Available from: http://www.ncbi. nlm.nih.gov/pmc/articles/PMC3977690/ 
17. Raaijmakers LGM, Hamers FJM, Martens MK, Bagchus C, Vries NK, Kremers SPJ. Perceived facilitators and barriers in diabetes care: a qualitative study among health care professionals in the Netherlands. BMC Fam Pract [internet]. 2013[cited 2015 Mar. 13]; 14(1):114. Available from:http://www.ncbi.nlm.nih.gov/pmc/ articles/PMC3751909/

18. Faria HTG, Veras VS, Xavier ATFX, Teixeira CRS, Zanetti ML, Santos MA. Quality of life in patients with diabetes mellitus before and after their participation in an educational program. Rev Esc Enferm USP. 2013; 47(2):354-8.
19. Ratanawongsa N, Bandhari VK, Handley $M$, Rundall T, Hammer H, Schilinger D. Primary care provider perceptions of the effectiveness of two self-management support programs for vulnerable patients with diabetes. J Diabetes Sci Technol. 2012; 6(1):116-24. 